\title{
El desarrollo de la investigación científica en los centros regionales desde los centros regionales
}

\author{
Kevin Estévez ${ }^{1}$ \\ Mirta Macías ${ }^{2}$ \\ German Hernández ${ }^{3}$ \\ Carlos Aceituno ${ }^{4}$
}

\section{CT/ Háblenos sobre la importancia de actividades como el encuentro anual de} coordinadores.

$\mathrm{KE} /$ Es un encuentro que a nosotros como coordinadores nos sirve para ver las experiencias que los otros compañeros han tenido en sus centros regionales $y$, si bien es cierto, cada centro tiene su contexto, la mayoría de problemas a los cuales nos enfrentamos son muy comunes. Este encuentro nos sirve para encontrar posibles salidas a esos problemas y con la ayuda de la Dirección de Investigación formar planes de trabajo para poder salir de este tipo de problemática con que todos los días nos encontramos en las coordinaciones regionales.

$\mathrm{MM}$ / Es como una radiografía de lo que cada centro regional está haciendo en materia de investigación, ahí vemos cuáles son las fortalezas de uno y en qué debemos mejorar.

GH/ Los encuentros significan para nosotros un espacio de reflexión, no solamente para reflexionar sobre lo que estamos haciendo sino también para considerar todos aquellos aspectos fuertes que tienen otros centros que nos pueden servir a nosotros para impulsar el trabajo que estamos desarrollando. Un hecho muy importante es ver la experiencia de los demás centros regionales donde vemos el punto de atención y la fortaleza, y nos permite ver que si esa fortaleza en nosotros es una debilidad, puede ser un elemento que nos motive y que nos ayude a superar o alcanzar un nivel de mayor desarrollo como centro regional.

CA/ Nos ha ayudado bastante con las ideas que nos ha proporcionado el asistir, porque hemos asistido algunos profesores, hemos traído estudiantes y nos ha

${ }^{1}$ Coordinador regional del Centro Universitario Regional de Occidente, CUROC

2 Coordinadora regional del Valle de Sula, UNAH-VS

${ }^{3}$ Coordinador regional del Centro Universitario Regional del Centro, CURC

${ }^{4}$ Coordinador regional del Centro Universitario Regional Litoral Pacífico, CURLP 
contribuido a que las líneas de investigación se fortalezcan un poco más. El conocimiento de las ponencias, de las presentaciones que se hacen en los proyectos nos da apertura para nuevos programas de investigación, nos da ideas, nos fortalece para esos nuevos proyectos, ya sea en el área de agroindustria, acuícola, economía, todo está relacionado, porque tenemos el tiempo para disfrutar en el congreso y no solo estar a lo que compete en nuestra área, sino que nos da tiempo para llegar a otras ponencias de relevancia.

\section{$\mathrm{CT} /$ ¿Cuáles son las expectativas frente a este XI Congreso?}

$\mathrm{KE} /$ Las expectativas nuestras como CUROC son grandes porque venimos participando desde 2013 en este tipo de congresos, recuerdo que 2013 solo era una ponencia la que presentamos y hemos venido creciendo año con año, traemos más conferencias, más profesores se involucran al ver el resultado que han obtenido otros copmañeros y eso nos hace tener una participación mayor porque hay más investigaciones que se han ejecutado a lo largo del año y eso nos permite venir a presentar resultados al congreso.

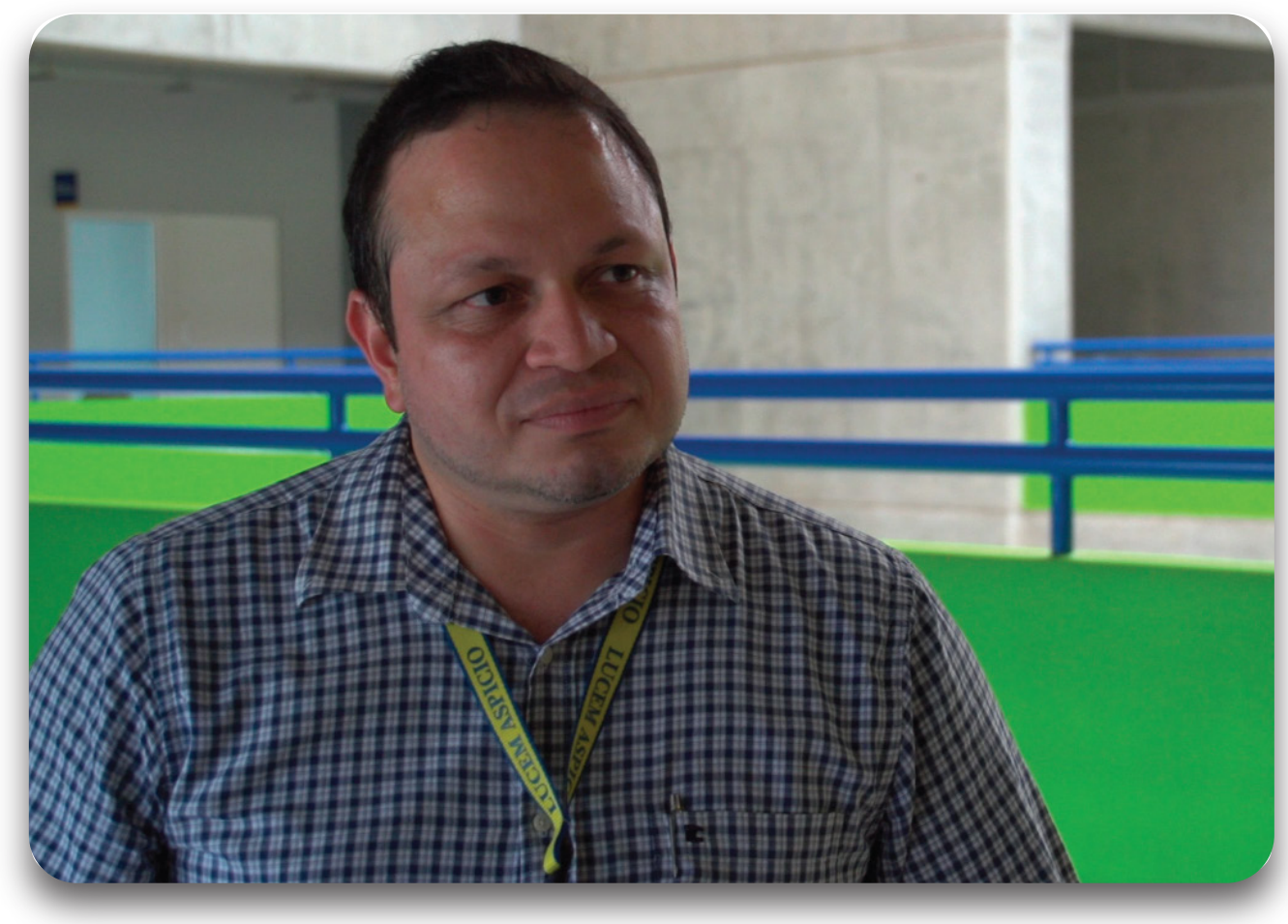

Kevin Estévez 
CA/ Como Centro regional queremos tener una diversidad de proyectos y dentro de esta diversidad, estamos considerando desde el punto de vista de la carrerade ingeniería acuícola, como de la carrera de agroindustria. Dentro de estos componebntes incluye la participación de profesores investigadores, como tenemos para este congreso lo que es estudiantes que están participando y que desarrollaron proyectos de investigación y que estarán haciendo ponencias en el próximo congreso.

\section{$\mathrm{CT} /$ ¿Qué impacto ha tenido y cómo se percibe el congreso de investigación científica en el centro regional?}

$\mathrm{KE} /$ Viene a ser para nosotros como la culminación de todo un proceso de investigaciones que se desarrollan gracias al programa de becas que la dirección de investigación tiene y en la cual continuamente hemos estado participando y ganando fondos para ejecutar esas investigaciones. Para los profesores es como culminar con un proceso que incluye también la publicación de un artículo, pero la socialización en un congreso es muy importante para dar a conocer los resultados y para que otros investigadores también conozcan lo que hemos hecho y podamos hacer posteriormente otros proyectos en común. Siempre y cuando las temáticas sean de interés para ambas partes.

CA/ El impacto que tendría y realmente que es la esperanza que tenemos es un impacto de tremenda importancia, porque la zona sur de Honduras, donde está ubicado el CURLP, está bien comprometida y relacionada con la explotación pesquera. si la explotación pesquera se menciona que es un recurso que está a su mínimo, agotada, creo que el mejor aporte que podemos hacer desde la academia, la investigación y la vinculación, es hacia la reproducción de las especies marinas y contribuir a la repoblación de las especies en el golfo de fonseca.

\section{CT/ ¿Cuáles son los logros significativos que usted podría destacar del centro regionalen investigación en 2016 ?}

KE/ Nosotros hemos estado trabajando fuertemente en la estructura del Sistema de Investigación Científica y Tecnológica y actualmente nos estamos preparando para presentar la acreditación de dos unidades de gestión de investigación, de un observatorio universitario de mercado de trabajo que es una iniciativa en conjunto con la agencia de desarrollo estratégico local de Santa Rosa de Copán y la creación de un grupo de investigación en ingeniería de bioprocesos, que eso nos va a permitir tener la estructura necesaria para finalmente conformar nuestro 
Consejo General de Investigación Regional, y es un logro importante porque nosotros tal vez empezamos muy fuerte con becas de investigación pero habíamos descuidado la estructura. Entonces ahora estamos retomando ello para que este sistema se vuelva más fuerte en el centro regional.

MM/ Es la consolidación del Congreso de Investigación, que a partir de 2014 vimos que distintos actores tanto de la comunidad de Valle de Sula como internacionales dicen presente sin que nosotros los busquemos. Dentro de los colaboradores está el doctor Salvador Moncada que siempre dice presente al congreso y es uno de los logros, tenemos patrocinadores que siempre nos dicen presente y una vez finalizado, a partir de noviembre, ya nos mandan que quieren seguir promocionando o patrocinando el congreso.

GH/ Podríamos decir que los más representativo está en el hecho de dos líneas. Una que es el hecho de haber lgorado mayor formación en aspectos investigativos con respecto a los docentes. El otro aspecto es relacionado con los estudiantes donde también a través de ejercicios hemos desarrollado algunas capacidades unificando criterios con los docentes que enseñan investigación. El hecho de que

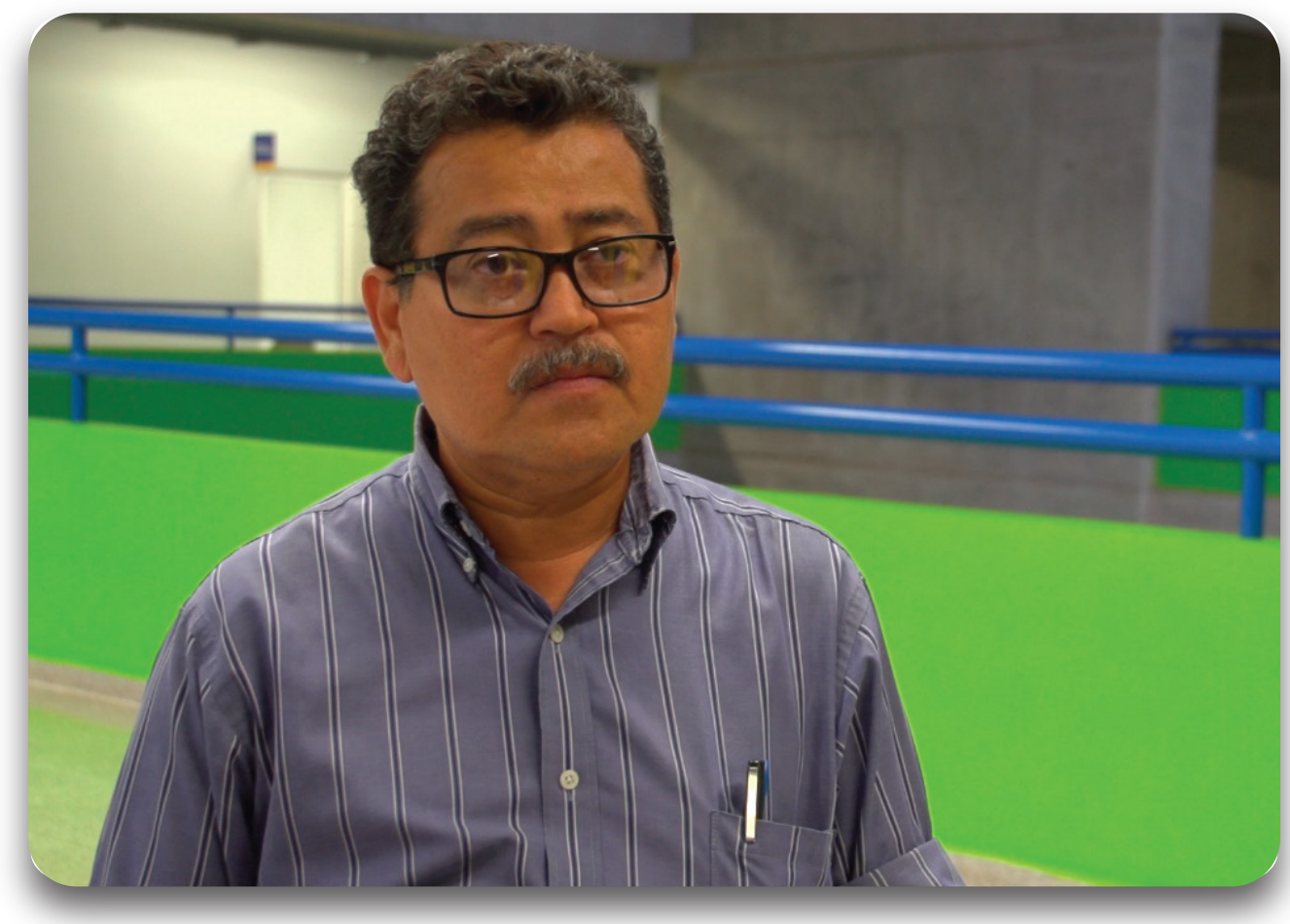

German Hernández 
los docentes desarrollen ciertas capacidades nos permite generalizar esos espacios de aprendizaje para los alumnos. Eso quiere decir que hay elementos básicos que estamos generalizando, como las metodologías, uso de un programa para trabajar datos, saber cómo estructurar un informe y que esto se convierta en una generalidad, ya no solamente para las asignaturas de investigación, sino también estamos influyendo en las otras asignaturas.

\section{CT/ ¿Qué propone para mejorar la gestión de la investigación en el centro regional?}

KE/ Creo que debe haber un acercamiento mucho mayor a nuestros jefes de departamento, a coordinadores de carrera e incluso a nuestros profesores y estudiantes para que ellos, cada vez se vayan involucrando más personas en estos procesos, que vean a través de los buenos resultados que se han alcanzado en algunas áreas, a otras áreas les llame la atención esto y ellos puedan involucrarse también. Nuestro reto grande es que cada vez más personas se vayan involucrando en estos procesos sin descuidar la califdasd de este tipo de investigaciones y de todo lo que podamos hacer para apoyar a los profesores ahí estaremos.

MM/ Se requiere un trabajo coordinado y voluntad de determinadas unidades, porque hay ciertas limitantes, pero en la medida que se vayan empoderando, que haya más difusión, se vean los resultados, todos vamos entrando a una dinámica, porque es un esfuerzo compartido.

GH/ La gestión tiene que ser un poco más, la recomendación que se nos ha hecho, el tener un consejo regional, que nos permitiría definir claramente esas políticas a nivel institucional y hacer que prevalezca el nivel institucional como un elemento necesario. Si no se retoma a veces como un elemento de un grupo interesado o de una persona que está interesada, pero cuando llega a una estructura fundamental de centro regional eso permite mayor fortaleza, porque ahora hay una inclusión directa, no es solamente mediante una pretensión, sino el hehco de que las autoridades toman un papel directo en ello.

\section{CT/ ¿El avance de la investigación en los posgrados del centro regional?}

$\mathrm{KE} /$ Por el momento existe un posgrado en formulación y evaluación de proyectos, pero hay otros en gestión, como uno en agricultura sostenible y cambio climático, y esperamos que exista una ampliación del posgrado en seguridad alimentaria que se sirve en Comayagua, pero obviamente esto va a traer un gran impulso de 


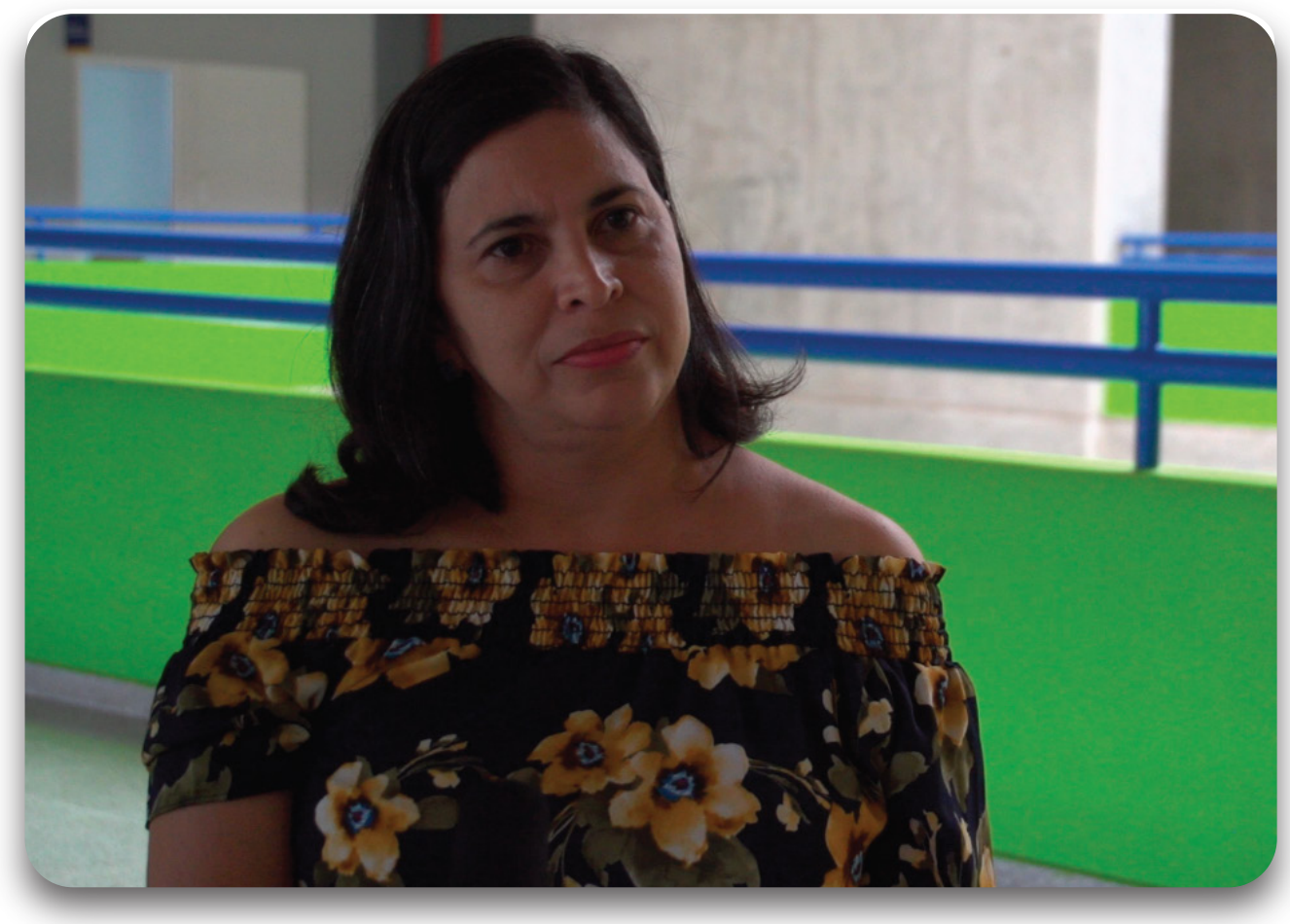

Mirta Macías

la investigación, porque si hay 25 participantes de posgrado, son 25 potenciales temas que puede haber y nosotros como parte de la coordinación regional estamos para ayudarle al menos en el aspecto metodológico que se necesita en estos casos.

MM/ En la medida que se consolide, creo que vamos a lograr mejores alianzas estratégicas, porque muchas veces llegan con proyectos de investigación y no tenemos el investigador, pero este proceso de consolidación ha sido paulatino y la sociedad de Valle de Sula se ha ido enterando, dicen presente, colaboran, hacen alianzas estratégicas para determinadas áreas de investigación.

$\mathrm{CT} /$ ¿De qué manera tratan de visibilizar los resultados de los proyectos de investigación en publicaciones o congresos?

KE/ Nosotros ocupamos todos los medios que la Dirección de Investigación pone a nuestra disposición, ya sea el congreso, las revistas Portal de la Ciencia para estudiantes y Ciencia y Tecnología para docentes, pero sentimos la necesidad de dar a conocer a través de otros medios los resultados de las investigaciones, 
principalmente para que tengan un impacto en la sociedad. Actualmente nosotros, al finalizar cada ivnestigación que se hace en el centro, hacemos reuniones de socialización donde se invita a otros profesores, incluso de otras universidade,s estudiantes, actores sociales, la población que está involucrada en los estudios y de esa manera buscamos tener un impacto en las personas que tienen poder de decisión y nosotros al tratar un problema dentro de la investigación dar una posible solución.

MM/ Les estamos dando salida a través de las revistas de la UNAH, tanto Portal de la Ciencia como Ciencia y Tecnología. Nosotros tenemos una propia memoria del congreso donde van todos los abstract de las propuestas, queremos hacer artículos a partir del congreso, un primer volumen que visibilice los resultados de investigación. Los congresos forman parte de esa visbilización de los resultados a nivel institucional.

GH/ Creo que en primer lugar el hecho de que nosotros los resultados los socializamos a nivel de la sociedad, no solamente a nivel del aula, sino que hacemos eventos en la ciudad, en un centro de cultura o en un hotel para que se presenten

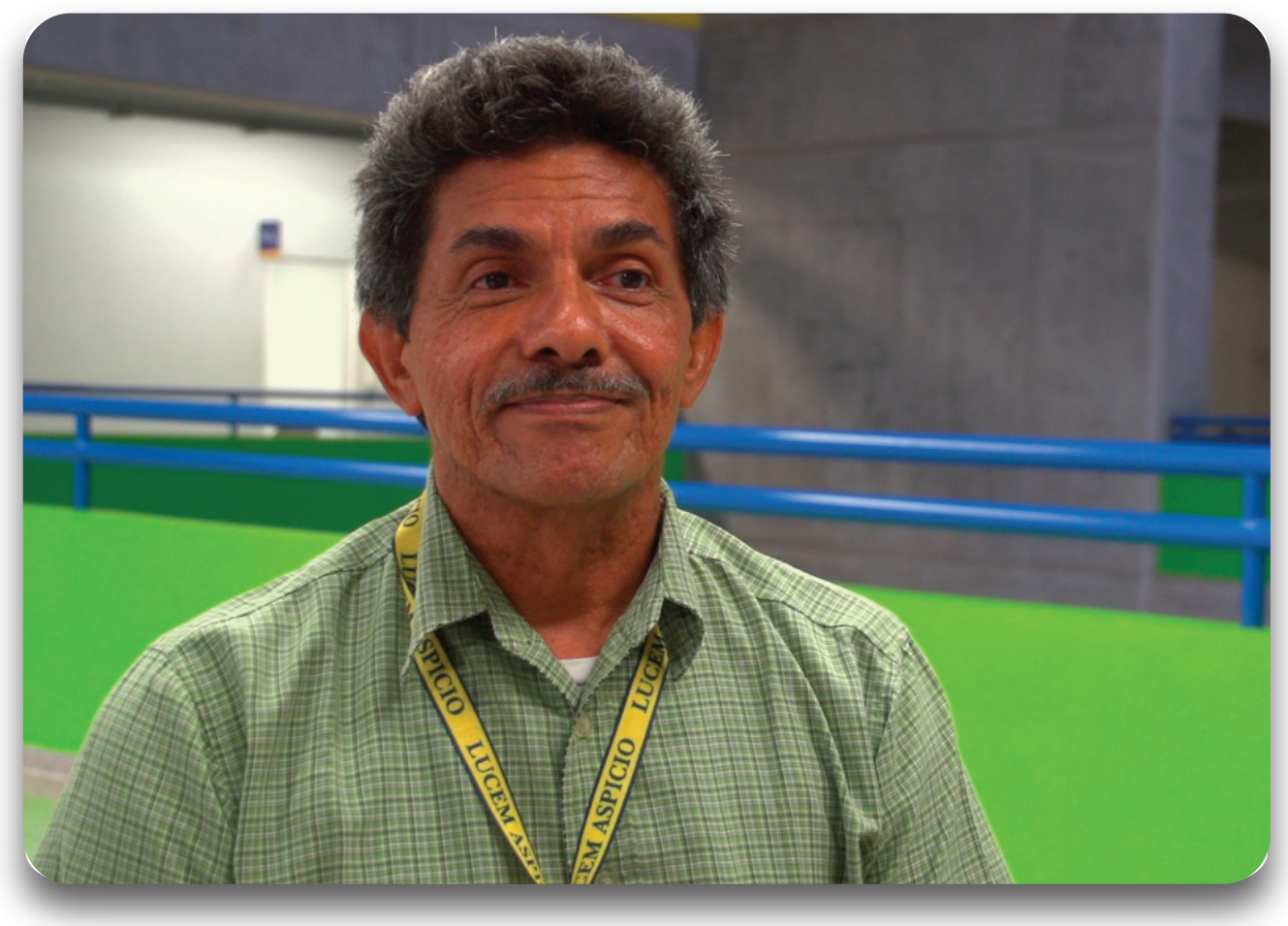

Carlos Aceituno 
los resultados y se llama a las autoridades que están involucradas con los temas. Estamos visualizando mayor efectividad y devolverle a la sociedad. otro elemento que utilizamos es que las instituciones están creyendo en nosotros, en que nosotros podemos ayudarles a generar espacios de investigación propias para que puedan desarrollar actividades o tomar decisiones eso nos vuelve más inclusivo. otro aspecto es el hecho de estar participando a nivel social con los actores que nos permitan darle a la sociedad algún punto de vista crítico, analítico a través de los estudios proponiendo algunas alternativas para el mejoramiento de tipo social. Esto no solamente a nivel municipal, sino incluimos procesos de salud, porque a veces nos invitan y hemos desarrollado conversatorios para hacer procesos más efectivos. 